\title{
Aplicación de RCM en equipo de esterilizado de acuerdo a la Gestión de Riesgos
}

\section{RCM application in sterilized equipment according to Risk Management}

\author{
MENDEZ-GOVEA, Luis Alberto \& TUDÓN-MARTÍNEZ, Alberto
}

Universidad Tecnológica de San Luis Potosí, Av. Dr. Arturo Nava Jaimes 100, San Luis Potosí, SLP. C.P. 78430

ID $1^{\mathrm{er}}$ Autor: Luis Alberto, Méndez-Govea / ORC ID: 0000-0002-9763-2519, Researcher ID Thomson: X-7378-2019, CVU CONACYT ID: 1014457

ID $1^{\text {er }}$ Coautor: Alberto, Tudón-Martínez / ORC ID: 0000-0003-1689-1250, CVU CONACYT ID: 411753

DOI: $10.35429 /$ JIE.2019.8.3.1.5

Recibido 25 de Abril, 2019; Aceptado 02 Junio, 2019

\section{Resumen}

En el siguiente artículo se describe la aplicación del RCM (Mantenimiento Centrado en Confiabilidad) con base a la Gestión de Riesgos, en un equipo de Esterilizado en una empresa del ramo alimenticio. La necesidad de aplicar el RCM, es debido a que el equipo de esterilizado presentaba una Disponibilidad de equipo muy baja, así como un aumento en los costos de mantenimiento, en merma de materiales y materia prima, además de afectar a la calidad e inocuidad del producto esterilizado. El equipo de esterilizado se fabrícó en 1955 , ya con el paso del tiempo, la mayor parte de sus componentes metálicos internos presentaban un alto índice de corrosión, generando una mala sincronización, dañando el producto a esterilizar. Debido a estos problemas, constantemente se tenían incumplimientos en los programas de producción, así como en las entregas hacia los clientes.

RCM, Gestión de Riesgos RCM, Aplicación de RCM

\begin{abstract}
The following article describes the application of the RCM (Reliability Centered Maintenance) according to Risk Management, in a Sterilized equipment in a food business. The need to apply the RCM, is due to the fact that the Sterilized equipment had a very low availability of equipment, as well as an increase in maintenance costs, in the scrap of materials, in addition to affecting the quality and safety of the sterilized product. The sterilizing equipment was manufactured in 1955, and with the passage of time, most of its internal metal components had a high rate of corrosion, generating a poor synchronization, damaging the product to be sterilized. Due to these problems, there were constantly defaults in production programs, as well as deliveries to customers
\end{abstract}

RCM, RCM risk management, RCM application

Citación: MENDEZ-GOVEA, Luis Alberto \& TUDÓN-MARTÍNEZ, Alberto. Aplicación de RCM en equipo de esterilizado de acuerdo a la Gestión de Riesgos. Revista de Ingeniería Industrial. 2019. 3-8: 1-5

\footnotetext{
*Correspondencia al Autor (Correo Electrónico:1mendez@utslp.edu.mx)

$\dagger$ Investigador contribuyendo como primer autor.
} 


\section{Introducción}

Con la actualización de la norma ISO 9001:2015 e IATF 16949:2016, se tiene un nuevo esquema que es la Gestión de Riesgos, en donde se deben determinar los riesgos y oportunidades (Standarization, Norma ISO 9001:2015, 2015), para asegurar que el Sistema de Gestión de Calidad logre sus objetivos, aumente los defectos deseables y prevenga o reduzca los efectos no deseados.

RCM (Moubray, 2004), Mantenimiento Centrado en Confiabilidad, permite identificar las políticas que deben ser implementadas para manejar los modos de falla que pudieran causar las fallas funcionales de cualquier activo físico en un contexto operacional dado.

\section{Descripción del problema}

Se contaba con una máquina de esterilizado para producto enlatado en el ramo almenticio, la cual se fabricó en el año 1955, la empresa fabricante del equipo era de USA, sin embargo, en esos momentos no se contaba con sus datos para poder contactarlo.

Cuenta con dos etapas: Una de calentamiento y otra de enfriamiento, debido a la naturaleza de su operación las condiciones internas de la máquina están expuestas a la humedad y temperaturas desde $100{ }^{\circ} \mathrm{C}$ hasta $34^{\circ} \mathrm{C}$, con el paso del tiempo se fue incrementando la corrosión en sus componentes.

Los principales problemas generados por sus condiciones físicas son: el aumento en los costos del mantenimiento, en scrap de materia prima y materiales, incumplimientos en las entregas del producto terminado al cliente, tiempo muerto mensual promedio de 2,192 minutos, sumando una pérdida total de USD $\$ 700,000$ en un período de 1.5 años.

\section{Objetivo}

Con base a los resultados obtenidos, se define como objetivo, tener por lo menos una reducción del $50 \%$ en los tiempos de paro no programados (tiempos muertos) en la máquina de esterilizado.

\section{Metodología}

Para lograr el objetivo establecido, se selecciona la técnica RCM para identificar los riesgos y aumentar la confiabilidad de la máquina.

RCM (Reliability Centered Maintenance) está regido por las normas SAE JA1011 (International, SAE JA1011, 1999) y SAE JA1012 (International, SAE JA1012, 2002).

El RCM es una técnica recomendada por ISO 31010 (Standarization, ISO 31010, 2009) para realizar la evaluación de riesgos, la cual permite al departamento de Mantenimiento e Ingeniería identificar los riesgos y oportunidades en la maquinaria y equipo.

Otra técnica utilizada en conjunto con RCM, es el AMEF (Análisis de Modo y Efecto del Fallo). El AMEF (AIAG, 2008) permite identificar en la maquinaria: Las funciones operacionales, los fallos de la función, los modos y efectos de los fallos, los cuales permiten entender qué es lo que sucede en el contexto de la operación de la maquinaria.

\section{Contribución de la investigación}

Una contribución importante de éste artículo, es que el personal de mantenimiento se mantenga actualizado en la aplicación de las diferentes técnicas que ayudan a mejorar los Kpi's de su proceso, con base a la normatividad ISO actual.

También, que constantemente estén trabajando en la mejora continua, bajo un Sistema de Gestión de Mantenimiento de clase mundial y no con el sistema tradicional de mantenimiento.

\section{Desarrollo del RCM}

Durante la aplicación del RCM, se llevaron a cabo las siguientes etapas:

1. Formación de equipo de trabajo

2. Aplicación del RCM

3. Resultados obtenidos

4. Contribución

5. Sugerencias del tema

6. Referencias 


\section{Formación de equipo de trabajo}

Un factor importante para el desarrollo de los talleres de RCM, es el involucramiento del personal relacionado con la maquinaria a analizar.

Para tener una mejor apertura en la identificación de las áreas de oportunidad, se formó un equipo multidisciplinario (Figura 1) integrado por:

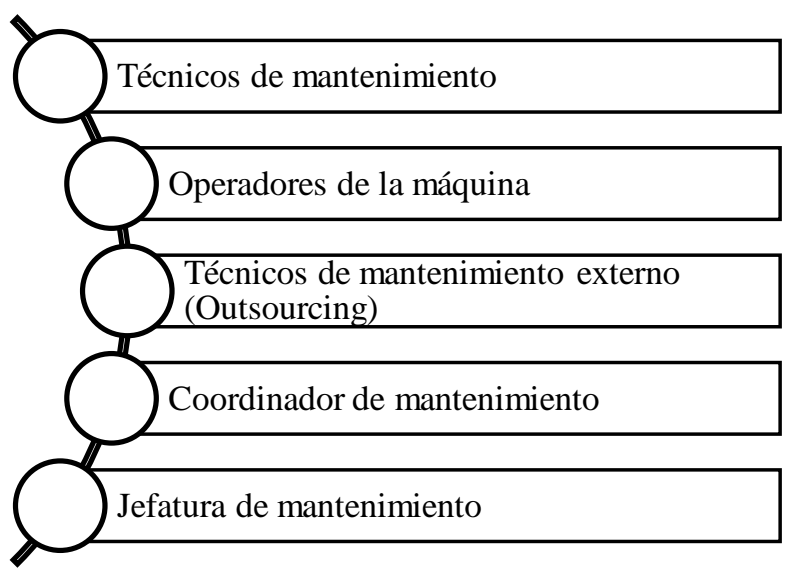

Figura 1 Equipo multidisciplinario

\section{Aplicación del RCM}

Una vez formado el equipo de trabajo RCM, se programaron sesiones de trabajo, se les dio una retroalimentación a los integrantes del equipo, sobre las condiciones físicas de la máquina de esterilizado, así como la situación actual en los resultados operativos.

Como primer paso, con base a la estructura del diseño, se definieron los sistemas de operación de la máquina de esterilizado, para identificar de una manera más eficiente el flujo de su proceso operativo (Figura 2), tales como:

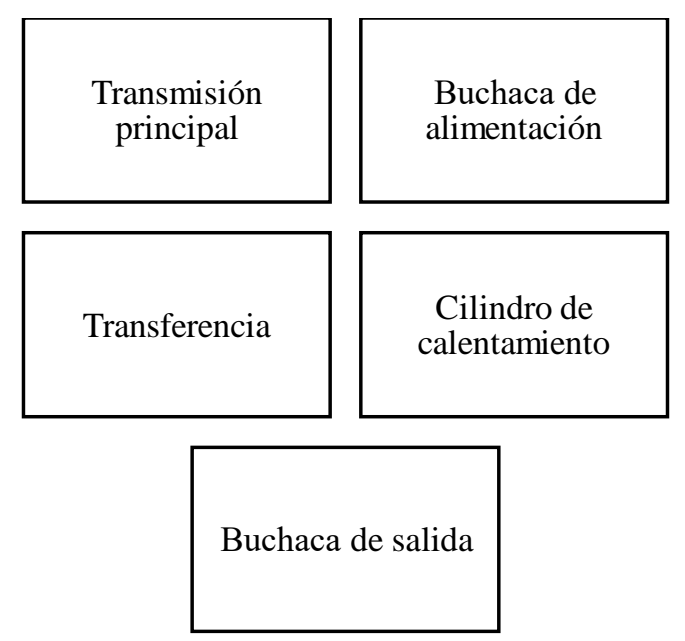

Figura 2 Sistemas de operación máquina de esterlilizado
Como apoyo de la técnica RCM, se utiliza el AMEF (Análisis de Modo y Efecto del Fallo), que permite identificar: Las funciones operacionales, los fallos de la función, los modos y efectos de los fallos, a continuación se muestra un extracto de los mismos.

Se identificaron las funciones operativas de cada una de los sistemas de la máquina, con la aportación de los conocimientos del equipo multidisciplinario (Figura 3), se pueden mencionar:

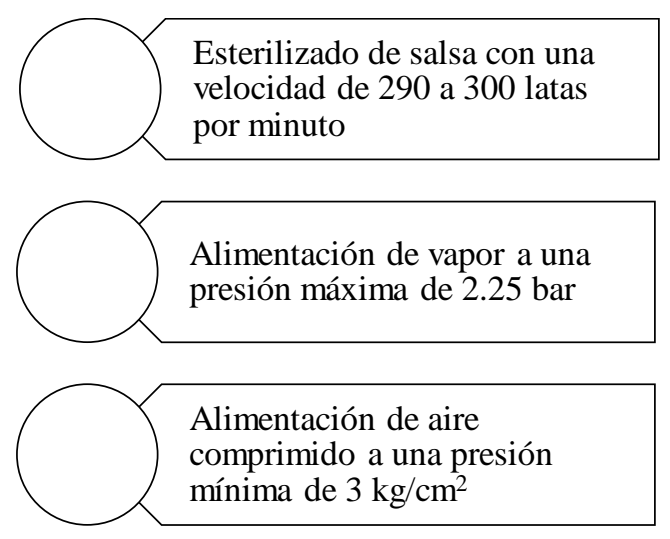

Figura 3 Funciones operativas

Como siguiente paso se inició con la identificación de los modos de fallo de las funciones operacionales (Figura 4), tales como:

\begin{tabular}{|c|c|c|}
\hline $\begin{array}{l}\text { Transmisión } \\
\text { principal }\end{array}$ & $\begin{array}{l}\text { Buchaca de } \\
\text { alimentación }\end{array}$ & Transferencia \\
\hline $\begin{array}{l}\text { - Motor } \\
\text { dañado } \\
\text { - Catarinas y } \\
\text { cadenas } \\
\text { dañadas } \\
\text { - Chumacera } \\
\text { con falta de } \\
\text { lubricante }\end{array}$ & $\begin{array}{l}\text { - Cadena de } \\
\text { alimentación } \\
\text { desalineada } \\
\text { - Bote volteado } \\
\text {-Exceso de } \\
\text { suciedad }\end{array}$ & $\begin{array}{l}\text { - Fuera de } \\
\text { tiempo } \\
\text { - Ángulos } \\
\text { doblados }\end{array}$ \\
\hline
\end{tabular}

Figura 4 Modos de Fallo

Cada vez que se presenta un modo de fallo, se generan diferentes consecuencias que generalmente no se consideran durante el contexto de la operación de las máquinas.

Debido a esto, se evalúan los efectos de las fallas (Figura 5), donde los integrantes del equipo, con base a su experiencia, definen las consecuencias operativas, se consideran los efectos que han sucedido con el paso del tiempo, tomando en cuenta también los que aún no se han presentado, algunos efectos identificados son: 


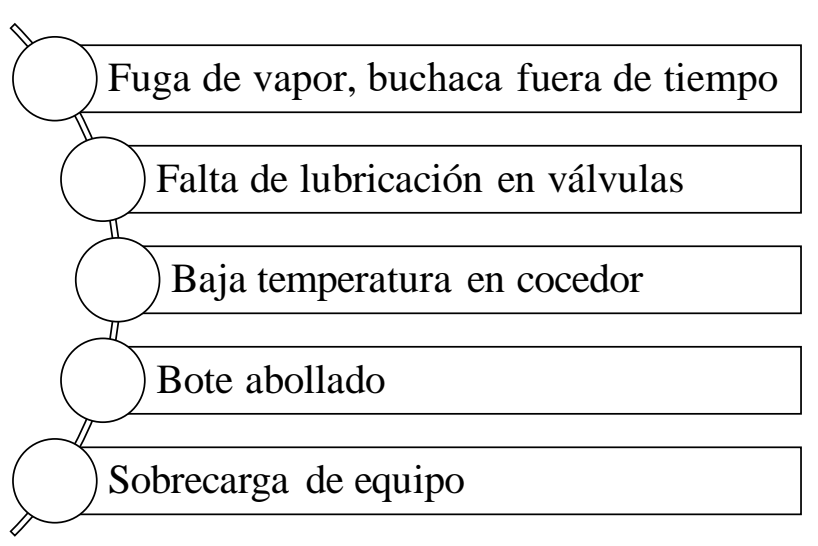

Figura 5 Efectos del Fallo

En la aplicación del AMEF de maquinaria, los integrantes del equipo participan, aportando sus ideas y mantienen la mente abierta, ya que cualquier comentario puede ser crucial para identificar los riesgos y oportunidades.

Una vez concluido el AMEF de maquinaria, el siguiente paso de acuerdo con la técnica RCM, es verificar cómo se pueden identificar y controlar los modos de fallo, de acuerdo al contexto de la operación, con base a las consecuencias ocultas, a la seguridad, medio ambiente, operacional y no operacional.

Finalmente se obtienen las diferentes actividades de mantenimiento preventivo y predictivo basadas en la confiabilidad, podemos mencionar las siguientes en la Tabla 1.

\section{Resultados obtenidos}

Durante el desarrollo de las técnicas de RCM y AMEF, se puede mencionar que como resultado, el personal que estuvo involucrado en su aplicación, entendió realmente qué es lo que sucede con el contexto de la operación de la máquina.

Esto permite que el personal pueda tomar decisiones más asertivas, minimizando las consecuencias de los riesgos negativos y aumentando los efectos de las consecuencias en los riesgos positivos. Se identificaron un total de 141 actividades para mantenimiento basado en confiabilidad (Gráfica 1).

\begin{tabular}{||l|l|l|l|}
\hline \multicolumn{1}{|c}{ Tareas propuestas } & \multicolumn{1}{c}{$\begin{array}{c}\text { Tipo de } \\
\text { mantenimiento }\end{array}$} & \multicolumn{1}{c|}{ Frecuencia } & Responsable \\
\hline $\begin{array}{l}\text { Medir } \\
\text { aislamiento de } \\
\text { motor eléctrico }\end{array}$ & Predictivo & Semestral & $\begin{array}{l}\text { Tec. } \\
\text { Eléctrico }\end{array}$ \\
\hline $\begin{array}{l}\text { Lubricación de } \\
\text { baleros }\end{array}$ & Preventivo & Mensual & $\begin{array}{l}\text { Tec. } \\
\text { Mécánico }\end{array}$ \\
\hline $\begin{array}{l}\text { Alineación y } \\
\text { limpieza de } \\
\text { catarinas y } \\
\text { cadenas }\end{array}$ & Preventivo & Trimestral & $\begin{array}{l}\text { Tec. } \\
\text { Mecánico }\end{array}$ \\
\hline $\begin{array}{l}\text { Inspección de } \\
\text { ruido anormales } \\
\text { en transmisión } \\
\text { de cadena }\end{array}$ & Autónomo & $\begin{array}{l}\text { Al inicio de } \\
\text { turno }\end{array}$ & Operador \\
\hline
\end{tabular}

Tabla 1 Tareas de Mantenimiento Preventivo basado en Confiabilidad

\section{Actividades por tipo de mantenimiento}

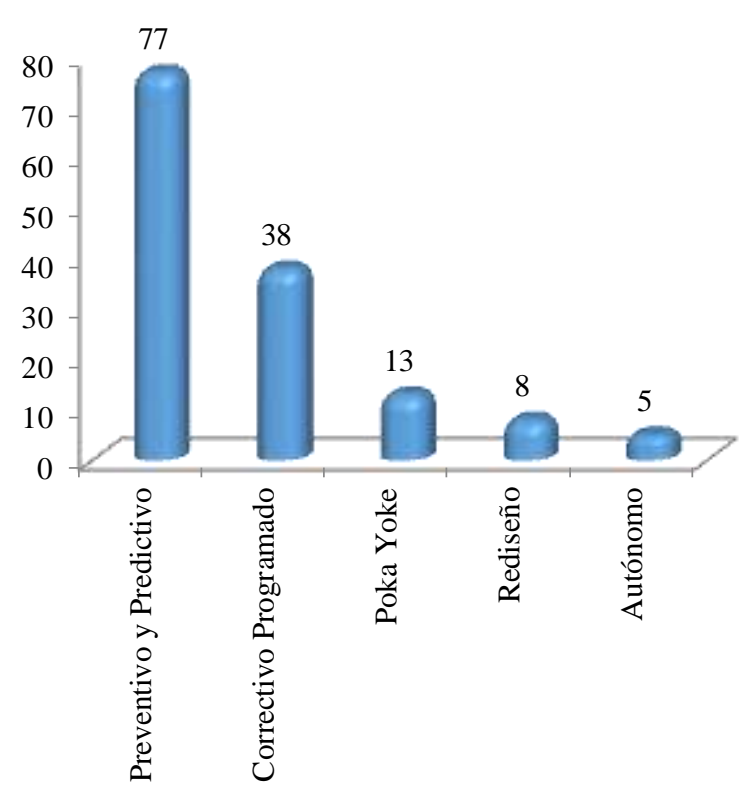

Gráfica 1 Actividades por tipo de mantenimiento máquina de esterilizado

Se obtuvo una reducción de tiempo muerto hasta de un $85 \%$ mensual del equipo de esterilizado (Gráfica 2).

\section{Tiempo muerto mensual (minutos)}

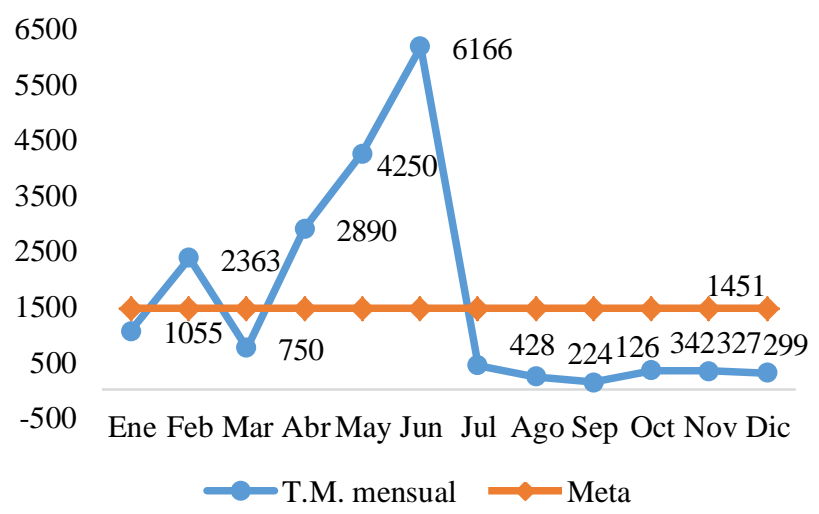

Gráfica 2 Tiempo muerto mensual del año, máquina de esterlilizado 
Los integrantes del equipo multidisciplinarios, comprendieron el contexto operativo de la máquina de esterlilizado

Aunque el equipo se fabricó en 1955, se pudo mantener controlado, posteriormente se generó un análisis costo beneficio resultado del $\mathrm{RCM}$, en donde se justifica el cambio de la máquina por otra nueva.

\section{Contribución}

La aplicación de la técnica RCM abre un panorama enfocado hacia cómo es el contexto operativo de la maquinaria y equipo.

La participación de un equipo multidisciplinario, incluyendo todos sus conocimientos y experiencia, enriquece la madurez de los participantes.

Se fomenta el trabajo en equipo y tomar en cuenta la opinión de todos los involucrados.

\section{Sugerencias del tema}

Algunas sugerencias para que sea exitoso el desarrollo de los talleres RCM son:

Los integrantes del equipo multidisciplinario tengan formación previa referente al RCM y el AMEF.

Que los procesos productivos tengan el esquema de equipos de trabajo multidisciplinarios.

Una base de datos confiable en el historial de mantenimiento de la maquinaria.

Respetar la programación de las sesiones de trabajo en RCM.

\section{Referencias}

AIAG, A. I. (2008). Potential Failure Mode and Effects Analysis (FMEA). 4th Edition.

International, S. (1999). SAE JA1011. Criterios de Evaluación para Procesos de Mantenimiento Centrado en Confiabilidad.

International, S. (2002). SAE JA1012. Guía para la Norma de Mantenimiento Centrado en Confiabilidad.
Moubray, J. (2004). RCM Mantenimiento Centrado en Confiabilidad. North Carolina, USA: Aladon LCC.

Standarization, I. O. (2009). ISO 31010. Gestión de Riesgos-Técnicas para Evaluación de Riesgos.

Standarization, I. O. (2015). Norma ISO 9001:2015. Sistema de Gestión de Calidad Requerimientos. 